Editorial

\title{
A Message from the editor-in-chief about progress in cancer prevention and current research
}

\section{Editorial}

Thanks to the efforts of our Editors, Associate Editors and to the contributing scientists, JCPCR has so far published more than two hundred manuscripts in areas of cancer prevention and current research. The journal is listed in several scientific search engines and hopes to be listed in Google Scholar in the near future. Still, we strive to accomplish more progress.

As the second decade of the $21^{\text {st }}$ Century starts to wrap up, our understanding of the complex biological aberrations associated with neoplasia and pre-neoplasia is unfolding at an accelerated phase. We hope that during the current year more is published on the molecular interaction between the external environment and the body regarding neoplastic transformation, progression, metastasis and immunity.

For a long time, epidemiologic studies had suggested that the majority of cancers are environmental in origin. Chronic infections, irradiation, UV light, smoking, high caloric intake and obesity are significant risk factors in cancer. These "external stimuli" and associated oxidative stress produce epigenetic modulation in various tissues affected. Genes associated with proliferation and angiogenesis are activated mostly by hypomethylation and suppressor genes are inactivated. ${ }^{1}$ At a systemic level, the immune system is suppressed mainly due to the pro-inflammatory cytokine response associated with these environmental stimuli. ${ }^{2}$ The end result is neoplastic transformation, progression and immune evasion. ${ }^{3}$

On the other hand, healthy life-style; exercise, low caloric diet, meditation, acupuncture...etc. stimulate the secretion of endorphin. This "internal" morphine contributes to a better mood and to the stimulation of the immune system especially NK cells and macrophages to attack cancer cells. ${ }^{4}$

Thus, cancer initiation and progression are in fact due to longterm interaction between the "external environment" and the "internal environment" including cytokines, hormones, neurotransmitters and the immune system. These culminate in the process of cellular proliferation and defective repair as well as a receptive microenvironment. A receptive microenvironment of immune cells, fibroblasts, vessels, macrophages and interstitial material supports tumor growth, invasion and metastasis.

Recent years witnessed a significant progress in cancer control with "targeted" therapy, hypomethylating drugs, check-point inhibition and immune modulation. We feel confident that the near future carries even more successes in combing hypomethylating drugs with other treatment modalities, including check-point inhibition. ${ }^{5}$
Volume 8 Issue 4 - 2017

Tahseen AI-Saleem

Department of Pathology, Temple University, USA

Correspondence: Tahseen AI-Saleem, Department of Pathology, Fox Chase Cancer Center, Temple University, Philadelphia, Pennsylvania, I 91 I I, USA, Tel (215)728-3850, Fax (2I5)728-2848; Emai I tahseen.al-saleem@fccc.edu

Received: August 22, 2017 | Published: August 31, 2017

Along these topics, results of research, reviews or editorials will be more than welcomed and expedited for publication.

\section{Acknowledgements}

None.

\section{Conflicts of interest}

The authors declare that there are no conflicts of interest.

\section{Funding}

None.

\section{References}

1. Hyndman IJ. Review: the Contribution of both Nature and Nurture to Carcinogenesis and Progression in Solid Tumours. 2016;9(1):63-69.

2. Obata Y, Furusawa Y, Hase K. Epigenetic modifications of the immune system in health and disease. I. 2015;93:226-232.

3. Alexandrov LB, Ju YS, Haase K, et al. Mutational signatures associated with tobacco smoking in human cancer. Science. 2016;354(6312):618622 .

4. Sarkar DK, Murugan S, Zhang C, et al. Regulation of cancer progression by beta-endorphin neuron. Cancer research. 2012;72(4):836-840.

5. Dear AE. Epigenetic Modulators and the New Immunotherapies. The New England journal of medicine. 2016;374(7):684-686. 